\title{
LA BRECHA DIGITAL COMO OBSTÁCULO AL DERECHO UNIVERSAL A LA EDUCACIÓN EN TIEMPOS DE PANDEMIA
}

\author{
THE DIGITAL DIVIDE AS AN OBSTACLE TO THE UNIVERSAL RIGHT TO \\ EDUCATION IN TIMES OF PANDEMIC
}

\author{
Ángel PRINCE TORRES ${ }^{1}$
}

\begin{tabular}{|lll|}
\hline Recibido & $:$ & 28.11 .2020 \\
Aceptado & $:$ & 21.12 .2020 \\
Publicado & $:$ & 12.02 .2021 \\
\hline
\end{tabular}

RESUMEN: En el mundo, la inequidad se hace palpable en distintos espacios de la sociedad bajo diferentes términos y con diversas variantes. Para abordar dicha circunstancia, este artículo tuvo como propósito analizar las consecuencias de la brecha digital como un obstáculo al acceso sobre el derecho universal a la educación en tiempos de pandemia. Debido a esto, se realizó un estudio de enfoque cualitativo y diseño documental con análisis de textos. Se determinó que la brecha digital es un factor que ha develado la falta de igualdad de ciertos grupos sociales para participar en el acto educativo debido a la falta de recursos tecnológicos, aun cuando existen normas jurídicas que defienden la equidad entre las personas. Se concluyó que, aunque ha existido incidencia de la COVID-19 y la brecha en el contexto educacional, representa la oportunidad para cambiar viejos esquemas sobre él y prepararse para futuras incidencias que pudieran acaecer.

Palabras clave: Brecha, tecnología, educación, derecho, pandemia.

ABSTRACT: Inequality is palpable in different spaces of society all around the world, under different terms and with different variants. To address this circumstance, this article aimed to analyze the impact of the digital divide as an obstacle to the access of the universal right to education in times of pandemic. Due to this, a study was structured with a qualitative approach, documentary design and text analysis. It was determined that the digital divide is a factor that has revealed the lack of equality of certain social groups to participate in the educational act due to lack of technological resources, even when there are legal rules that defend equality of the people. It was concluded that although there has been an incidence of COVID-19 and the digital divide in the educational context, it represents the opportunity to change old schemes about it and prepare for future incidents that may occur.

Keywords: Divide, technology, education, law, pandemic.

\footnotetext{
${ }^{1}$ Doctor en Ciencias de la Educación. Instituto Universitario Pedagógico “Monseñor Rafael Arias Blanco". Estado LaraRepública Bolivariana de Venezuela. Email: arbqto@gmail.com. ORCID: https://orcid.org/0000-0002-0059-7797
} 


\section{Journal of the Academy $|27|$}

\section{INTRODUCCIÓN}

Durante el año 2020 se ha realizado en gran parte del planeta un proceso de confinamiento como consecuencia de la expansión de la enfermedad por coronavirus COVID-19. Este hecho ha impactado no sólo los ámbitos político, económico y social, sino principalmente en la reconducción del proceso educativo abarcando los diferentes niveles implementados en gran variedad de territorios, pues tal como indican Pizan-Campos, Barros Sevillano y YupariAzabache (2020), se ha repercutido sobre las actividades presenciales y modificado los esquemas educacionales con potencial reducción del alcance de ellos hacia ciertos bloques de personas. Esto ha ocurrido visto que la Organización Mundial de la Salud caracterizó a la enfermedad como una pandemia, por lo cual la Organización Panamericana de la Salud (2020) consideró aclarar que:

La epidemia de COVID-19 fue declarada por la OMS una emergencia de salud pública de preocupación internacional el 30 de enero de 2020. La caracterización ahora de pandemia significa que la epidemia se ha extendido por varios países, continentes o todo el mundo, y que afecta a un gran número de personas (p.1)”.

En torno a lo anterior, el alcance de la enfermedad se considera como amplio, así que no solo refiere a una crisis de salud, sino también a una emergencia en todos los contextos de la vida cotidiana, pues muy bien se ha podido experimentar que no ha implicado únicamente la distorsión de diferentes libertades con las que cuenta el ser humano normalmente tales como la de asociación, desenvolvimiento de la personalidad o movilidad sin restricciones, por indicar algunos casos, sino que ha mantenido patrones que incidieron en la población adulta, adolescente e infantil. Por ello, se ha configurado la base ideal para atestiguar episodios de inequidad, visto que en estos tejidos las más afectadas siempre han sido aquellas personas que se encuentran en situación de vulnerabilidad económica, física o social y, de hecho, Santoro (citado por El Centro de Estudios en Salud y Sociedad, 2020) ha explicado en torno a esto que:

El efecto de la desigualdad social y las diferencias de clase y de capital (económico, pero también social, educativo, etc.), que van a generar consecuencias extremadamente dispares, no solo en tanto que son determinantes sociales de la salud, sino en las formas de enfrentarse a medidas como el cierre de escuelas o el fomento del teletrabajo y el elearning" (p. 21).

Así, tal como se nombra en el párrafo anterior, uno de los espacios que ha recibido gran carga en virtud de los acontecimientos sobre las incidencias del SARS-CoV-2, es el relativo a la 


\section{Journal of the Academy | $28 \mid$}

educación. El enfoque situacional ha implicado la toma de decisiones de los Estados hacia las maneras cómo conducir el proceso. También ocupa responsabilidad para establecer pautas de acción tanto a instituciones, como a los actores educativos que las conforman (docentes, personal administrativo, estudiantes, entre otros miembros de la comunidad educativa en general).

No es inadecuado tomar como válido entonces el criterio de Velásquez (2020), ya que teniendo en cuenta que para continuar con el aprendizaje en medio de la emergencia se optó mundialmente en términos generales por abrazar al acto educacional a distancia, la autora indica que “...los docentes no estaban acostumbrados a implementar dicha cuestión, cabe mencionar que las clases virtuales requiere preparación y formación previa, ya sea para impartir el curso de manera sincrónica o asincrónica” (Velásquez, 2020, p. 19).

Sin embargo, aparte de la preparación de los miembros de la comunidad educativa que habrían de participar de este hecho pedagógico y social, llevar a cabo la labor aquí indicada ha puesto sobre el tapete otra cuestión: la falta de equilibrio en la oportunidad de adherirse a los conocimientos desde el punto de vista del sistema educacional, cuando existe desigualdad en el acceso a los distintos espectros de las tecnologías de la comunicación e información (TIC). Esto, por lo tanto, implica también una conexión sobre la consideración de si las personas más desposeídas económicamente o vulnerables por razones culturales, geolocalización, entre otras, ven violentado de esta forma el ejercicio de uno de los derechos culturales más relevantes: el del acceso a la educación.

Para desarrollar el espectro de las cuestiones hasta aquí expresadas, cupo entonces realizar este trabajo cuyo propósito general fue analizar el impacto de la brecha digital como un obstáculo al acceso sobre el derecho a la educación en tiempos de pandemia. Del mismo modo, se establecieron propósitos específicos como: 1) Explicar el contenido de la brecha digital en el contexto de la pandemia; 2) Definir en qué consiste el derecho a la educación dentro de un marco universal y 3) Desglosar las consecuencias de la existencia de una brecha digital sobre el derecho humano a la educación. 


\section{Journal of the Academy $|29|$}

\section{DESARROLLO}

\section{Métodos y materiales}

Este estudio se configuró siguiendo al enfoque cualitativo para procesos investigativos, el cual de acuerdo con Sánchez (2019) "se sustenta en evidencias que se orientan más hacia la descripción profunda del fenómeno con la finalidad de comprenderlo y explicarlo" (p. 104), por lo que aquí se trata de revelar la vinculación entre la brecha digital evidenciada en tiempos de pandemia y el acceso al derecho fundamental a la educación. Por esta razón se implementó un diseño documental, en el cual, según Brito (2015) “[...] el investigador analiza los distintos fenómenos de la realidad obtenidos y registrados por otros investigadores en fuentes documentales" (p. 8).

Los recursos documentales chequeados consistieron en libros, artículos científicos en revistas académicas contenidas en bases de datos e índices como Redalyc, SciELO, entre otros como Google Scholar y en portales de internet pertenecientes a organizaciones y medios de reconocida solvencia informativa. Con ello se constituyó la recolección de datos provenientes de las fuentes secundarias prenombradas y establecidas a estos fines.

Para el análisis del material informativo ensamblado por medio de técnicas operacionales en el manejo de fuentes documentales (como el subrayado, la lectura en profundidad y el resumen), se usó al análisis crítico para establecer las relaciones de los contenidos. Dulzaides y Molina (2004), consideran que para la selección de textos pertinentes se deben tratar los documentos de acuerdo con el perfil de ellos y los elementos que los conforman, con lo cual puede posteriormente desglosarse la información a través de su escogencia y síntesis.

\section{La noción de la brecha digital y su relación con la pandemia}

Cuando se hace referencia a la brecha digital, también se realiza un llamado al concepto de desigualdad, pues en parte versa sobre los trances que vive parte de la población mundial para acceder a los servicios de conexión por intermedio de las tecnologías de la comunicación e información. En otras palabras, en vista que la brecha es asumida como la separación que puede existir entre un grupo y otro, no resulta extraño vincular ese concepto a la carestía en el alcance de los medios tecnológicos como canales de interconexión en un mundo globalizado. 


\section{Journal of the Academy $|30|$}

La concordancia entre la brecha digital y la desigualdad resulta ineludible, pues como aclaran Martin, Moore y Schindler (2016), esta última “[...] por lo general es definida por una combinación de indicadores económicos referidos a los ingresos y la riqueza. Sin embargo, es inseparable de diferencias sociales de otro tipo, una interdependencia que es particularmente evidente en el suministro de vivienda" (p. 30). Esta diferencia, como ya se ha dicho, también puede abarcar al alcance que se tiene en los espectros comunicacionales, los cuales resultan de gran trascendencia en el siglo XXI debido a que el ser humano cada día se ha hecho más dependiente del espectro tecnológico.

En el día a día de cualquier persona, no es extraño evidenciar que, en muchos casos, aquellos individuos que poseen más poder adquisitivo pueden costearse servicios de mejor calidad, tales como la internet. Esto representa un distanciamiento de la realidad que viven las personas que ni siquiera cuentan con los medios para pagarlo, o simplemente conviven en regiones donde no se asegura la conectividad. Sobre este punto, Alvarado, Gómez, Díaz y Martínez (2018) han aclarado que:

Inicialmente, la brecha digital se refería a la desigualdad entre aquellos que tenían o no tenían acceso físico a las tic. Pipa Norris fue una de las precursoras en la construcción del concepto al plantear distintos tipos de brecha: la brecha social, como la diferencia en el acceso a la información entre los pobres y ricos en cada país; la brecha global, como la diferencia entre países desarrollados y en desarrollo en el uso de tic; y la brecha democrática, como la diferencia entre quienes utilizan las tic para movilizarse y participar en la esfera pública (p. 51).

En otras palabras, la brecha digital abarca mucho más que solo el acceso a las comunicaciones, puesto que también cuando se evidencia, existe la representación de un indicador de la separación socioeconómica que existe en las distintas comunidades donde, aunque se propugne un nivel de igualdad incluso blindado a nivel jurídico, lamentablemente esto no se hace tangible del todo. Aparte, de acuerdo con Sánchez, Reyes, Ortiz y Olarte (2017), tal dispersión se vincula con la infraestructura tecnológica, pues también depende de cuán obsoletos sean los equipos (como los ordenadores) que fungen como recursos para el aprendizaje.

Estos hechos, tienen especial relevancia en el contexto de la pandemia, específicamente dentro del hecho educativo, porque como una solución a la necesidad de mantener una consecución en las actividades para el aprendizaje, se ha implementado de forma más amplia la educación 


\section{Journal of the Academy $|31|$}

a distancia para respetar las pautas del confinamiento social. Sobre esto, Mendoza (2020) ha aclarado que la implementación del aprendizaje remoto se ha establecido como alternativa forzosa en la ejecución de estrategias, porque a tenor de lo ocurrido con la COVID-19, de acuerdo con Organización de Naciones Unidas para la Educación, la Ciencia y la Cultura (UNESCO) un número mayor a 861.7 millones de jóvenes y niños en espacios de 119 países, han sido impactados por los efectos del aislamiento por el virus.

Por otra parte, en un nivel más maduro de desarrollo humano, Miguel (2020) incluyendo a la educación superior en los criterios de UNESCO, expresa que la pandemia ha afectado al segmento estudiantil en una proporción aproximada del $70 \%$ a nivel mundial. Ahora bien, el hecho de que gran cantidad de personas haya tenido que migrar desde la educación presencial o semi-presencial a la educación a distancia, ha hecho visible el espectro de desigualdades que hay al momento de contar con recursos tecnológicos para paliar este tipo de evento. El autor incluso se atreve a aclarar que la existencia de la brecha digital potencia la crisis en lugares como México (tan solo por ilustrar un ejemplo), donde para 2018, 46.5\% de las escuelas contaban con al menos un ordenador para actividades educacionales, siendo que, en 2019, un $56.5 \%$ de instituciones primarias y $57.9 \%$ de centros de enseñanza en secundaria tenían acceso a internet. Los números en los hogares no resultan muy distantes porque se computó el 56.4\% de ellos en donde se contaba con el prenombrado servicio.

En concordancia con lo anterior, es oportuno además complementar dando otro ejemplo sobre el contraste de oportunidades para el acceso educacional por medio de la virtualidad, pues la conectividad se enlaza en gran medida con el ancho de banda. Por ello, existen grandes medidas de separación en este sentido, como cuando Henríquez (2019) sostiene que al tiempo que Taiwán tiene internet de 85,02 megas, en Latinoamérica el país que más se le acerca es Uruguay con 9,2 megas y en territorios como Venezuela y Cuba están en 1,4 y 1,7 megas de velocidad. Asimismo, el Banco de Desarrollo de América Latina (2020) ha aclarado que "actualmente, 244 millones de latinoamericanos -el 38\% de la población- no tienen acceso a internet, una brecha social que ahonda la desigualdad en materia de acceso al conocimiento y oportunidades" (p. 1).

En otros lugares como el continente africano se ha determinado, tal como señalan García y González (2013) que "la tasa de penetración digital...es baja, y además presenta diferencias regionales relevantes, también si se comparan las zonas urbanas y las rurales. Es un reflejo, 


\section{Journal of the Academy | $32 \mid$}

cada vez más causa de la realidad social, política y económica del continente” (p. 128). De dicha manera se observa cómo la existencia de barreras en la comunicación de nueva generación, puede acrecentar los problemas sobre oportunidades para desarrollar actividades como las educacionales, y mucho más en épocas como la actual, en la cual se requiere dejar de lado temporalmente lo presencial para resguardar incluso la vida.

Las notas anteriores se ahondan no solo respecto a la falta de eficiencia en la prestación de servicios o la provisión de recursos tecnológicos, sino que es especialmente trascendental cuando existen lugares donde las personas ni siquiera cuentan con la posibilidad de acceder a ellos porque sencillamente no tienen electricidad, cables de conexión, entre otros. Esto ocurre en países como Ecuador, donde como alternativa para las poblaciones indígenas que no cuentan con las prenombradas herramientas, el Fondo Internacional de las Naciones Unidas para la Infancia (UNICEF, 2020) se ha encargado de repartir guías impresas para el autoaprendizaje en el contexto intercultural. Testimonios como el último de este párrafo denotan que existe un notable desequilibrio entre las posibilidades que tienen las personas que no pueden obtener ciertos insumos con respecto a otras que sí, lo cual se concatena con la siguiente afirmación:

En el contexto de la emergencia sanitaria, los niños, niñas y adolescentes con alguna discapacidad se han visto confinados a sus casas...La alternativa de la educación a distancia se convierte para muchos en un imposible... porque no tienen las destrezas o no están preparados en el uso de los dispositivos digitales, o porque los programas y actividades que los sistemas ofrecen a la mayoría no son accesibles para ellos [ ...]. Otro colectivo que ha salido especialmente perjudicado es el de los y las estudiantes de familias con menor nivel socioeconómico y dentro de ellos también los migrantes...Para los estudiantes de familias con más recursos, la educación se sigue produciendo, dado que viven en hogares cuyo capital sociocultural y mayores oportunidades tiende a mitigar el impacto en el aprendizaje y los efectos psicosociales que la interrupción de la educación presencial trae consigo (Duk y Murillo, 2020, p. 12).

En torno a todo lo hasta aquí esbozado, el resultado del proceso de recolección de información elaborado arroja entonces un hecho innegable: la pandemia ha generado una visualización de las desigualdades que en circunstancias normales tal vez pasan un poco más desapercibidas. Lo serio del asunto radica especialmente en que esa diferencia se manifiesta en el proceso enseñanza por intermedio de la aplicación de educación a distancia, ya que de acuerdo con Flores (2010), las comunidades para el aprendizaje desde recientes años se condicionan por los entornos virtuales, especialmente en ese tipo de mediación de conocimientos, y la brecha digital en distintos territorios ha dificultado el flujo correcto de desarrollo sobre las actividades 


\section{Journal of the Academy $|33|$}

pedagógicas y este es un punto que entonces se concatena con el ejercicio del derecho a una educación de calidad y oportuna.

\section{Sobre el derecho a la educación}

La educación, desde hace muchos años es considerada como un derecho humano, en tanto que es catalizador de condicionamiento en el desarrollo social. Además, se refiere a su caracterización dentro de esta esfera porque es una prerrogativa que no debería considerarse como un favor o una gracia por parte del Estado, sino que corresponde a niños, niñas, adolescentes, hombres y mujeres, por pertenecer a la especie humana, es decir, representa un natural reconocimiento de los mínimos esenciales que se deben asegurar a las personas para que logren el desarrollo de una vida digna.

El derecho a la educación se inserta dentro de la clasificación de los derechos económicos, sociales y culturales, los cuales conforme a Arias y Chaves (2010) son aquellos que se consideran de segunda generación en una clasificación de esta naturaleza. Estas prerrogativas se adhieren a los frutos de gestas revolucionarias de corte socialista y nacionalista a partir del siglo XX. Su principal sustento se encuentra en concepciones modernas ejecutables de manera particular, pero su titularidad tiene un carácter colectivo o individual, ya que versan sobre intereses jurídicos de alcance social.

Para Horbath y Gracia (2016) "el cumplimiento y derecho a la educación es de fundamental importancia pues soporta y se vincula con la posibilidad de acceder a otros derechos (trabajo, salud, vivienda, alimentación, entre otros)" (p. 173), y por ello debe notarse que esta consideración implica que dicha prerrogativa debería apartarse de parámetros discriminatorios, pues al tiempo que promueve la disminución de las brechas socioeconómicas a través de la apertura de oportunidades, del mismo modo debería ser disfrutada sin que existan otros elementos de separación que impidan el paso a ella. Del mismo modo, Muñoz (2014) estima a la educación desglosándola en los siguientes términos:

Los derechos humanos tutelan ética y jurídicamente el aprendizaje. Me refiero, por supuesto, a un derecho superior que no define los procesos vitales, sino que los protege y que, sin embargo, también consiste en un proceso cognitivo, pues implica una acción reflexiva y práctica sobre el quehacer cotidiano... 


\section{Journal of the Academy | $34 \mid$}

El aprendizaje es una actividad inevitable, o más bien, que al evitarse produce la extinción. Inevitable porque la acción conlleva al conocimiento y, viceversa, todo conocer es hacer (p. 3).

Con lo anterior en cuenta, puede afirmarse entonces que la educación constituye no solo un derecho, sino también un deber y corresponde a la necesidad que tienen las personas en desarrollarse tanto física como psíquicamente. Esto se circunscribe a que el acto educativo enriquece y es enriquecido por medio de las experiencias y los aprendizajes que se produzcan en la sociedad con el transcurrir del tiempo.

Por la importancia que estriba esta prerrogativa, se encuentra resguardada en instrumentos jurídicos internacionales que desarrollan el contenido de los derechos fundamentales. La Declaración Universal de los Derechos Humanos de la Organización de Naciones Unidas (ONU, 1948), estipula en su artículo 26 lo siguiente:

1. Toda persona tiene derecho a la educación. La educación debe ser gratuita, al menos en lo concerniente a la instrucción elemental y fundamental. La instrucción elemental será obligatoria. La instrucción técnica y profesional habrá de ser generalizada; el acceso a los estudios superiores será igual para todos, en función de los méritos respectivos. 2. La educación tendrá por objeto el pleno desarrollo de la personalidad humana y el fortalecimiento del respeto a los derechos humanos y a las libertades fundamentales; favorecerá la comprensión, la tolerancia y la amistad entre todas las naciones y todos los grupos étnicos o religiosos, y promoverá el desarrollo de las actividades de las Naciones Unidas para el mantenimiento de la paz. 3. Los padres tendrán derecho preferente a escoger el tipo de educación que habrá de darse a sus hijos (p. 6)

En la norma indicada, se establece entonces la gratuidad de la educación, su obligatoriedad, desarrollo en el marco de la no discriminación (nótese que indica en cuanto al nivel superior que será igual para todos, lo cual se encuentra comprometido cuando existen brechas que dificulten su realización), el libre desenvolvimiento de la personalidad en el seno de una sociedad fraterna e inclusiva y la libertad de asirse a algún tipo específico de esta prerrogativa. Por otra parte, el resguardo educativo también se produce en el Pacto Internacional de Derechos Económicos, Sociales y Culturales de la ONU (1966), cuando en su artículo 13 estatuye:

1. Los Estados Partes en el presente Pacto reconocen el derecho de toda persona a la educación. Convienen en que la educación debe orientarse hacia el pleno desarrollo de la personalidad humana y del sentido de su dignidad, y debe fortalecer el respeto por los derechos humanos y las libertades fundamentales. Convienen asimismo en que la educación debe capacitar a todas las personas para participar efectivamente en una 


\section{Journal of the Academy $|35|$}

sociedad libre, favorecer la comprensión, la tolerancia y la amistad entre todas las naciones y entre todos los grupos raciales, étnicos o religiosos, y promover las actividades de las Naciones Unidas en pro del mantenimiento de la paz.

2. Los Estados Partes en el presente Pacto reconocen que, con objeto de lograr el pleno ejercicio de este derecho: a) La enseñanza primaria debe ser obligatoria y asequible a todos gratuitamente; b) La enseñanza secundaria, en sus diferentes formas, incluso la enseñanza secundaria técnica y profesional, debe ser generalizada y hacerse accesible a todos, por cuantos medios sean apropiados, y en particular por la implantación progresiva de la enseñanza gratuita; c) La enseñanza superior debe hacerse igualmente accesible a todos, sobre la base de la capacidad de cada uno, por cuantos medios sean apropiados, y en particular por la implantación progresiva de la enseñanza gratuita; d) Debe fomentarse o intensificarse, en la medida de lo posible, la educación fundamental para aquellas personas que no hayan recibido o terminado el ciclo completo de instrucción primaria; e) Se debe proseguir activamente el desarrollo del sistema escolar en todos los ciclos de la enseñanza, implantar un sistema adecuado de becas, y mejorar continuamente las condiciones materiales del cuerpo docente" (p. 7).

En el Pacto, se reafirman y amplían los principios contenidos en la Declaración Universal de los Derechos Humanos, desarrollando especialmente el principio de gratuidad porque como ya se indicó, la educación es el medio a través del cual puede establecerse el avance social, pues esta es la finalidad última de la convivencia: el logro del desarrollo en la mayor cantidad de formas posibles. Es por ello que, de acuerdo a lo investigado, se estableció como resultado que la educación como un derecho constituye una herramienta para impulsar las capacidades personales, pero aparte se traduce en el marco de la obligatoriedad para garantizar incluso el logro de los objetivos estatales y comunitarios.

\section{Resultados y Discusión: El impacto de la brecha digital sobre el derecho a la educación}

Al ser este trabajo de naturaleza documental, el establecimiento de resultados se derivó de la pesquisa realizada, por lo que los datos suministrados constituyen el sustrato de las derivaciones consolidadas. De esta forma, se arrojaron como resultas las siguientes cuestiones:

a) Se determinó que las inequidades generadas por la brecha digital se encuentran interconectadas con brechas de otras clases, pues tan solo representa un indicador de demás elementos con los cuales no cuentan ciertos grupos sociales o culturales. Por ejemplo, denota el desequilibrio en el acceso a servicios, e igualmente puede indicar la diferencia económica entre personas.

b) Igualmente se pudo observar que la brecha digital no solo consiste en el acceso a las nuevas tecnologías a través de prestación de servicios por conectividad, sino que 


\section{Journal of the Academy | $36 \mid$}

incluye incluso aparatos y hardware utilizados para llevar a cabo la enseñanza a distancia.

c) La pandemia ha dado una revitalización a los mecanismos de aprendizaje a distancia.

d) La brecha digital condiciona el ejercicio del derecho a la educación.

e) El derecho a la educación es necesario para la consolidación de la estructura interna de los sujetos en el marco de los propósitos que la sociedad establece para una convivencia armónica.

f) El derecho a la educación es un derecho humano.

El derecho a la educación se observa como una prerrogativa preponderante, que se ha visto comprometida como consecuencia de la inequidad en el acceso a las tecnologías de la comunicación y la información. Dicho desequilibrio es sobre todo evidente cuando existe la contrastación de las relaciones antes expuestas en este trabajo, vinculadas con la capacidad para utilizar internet en territorios más desarrollados con respecto a aquellos en vías de desarrollo.

Ahora bien, una vez indicados los hallazgos, resulta conveniente establecer que la educación a distancia implementada como derivación de la enfermedad por coronavirus, conlleva algunas cuestiones que se alinean con las estipulaciones de Ruiz (2020), en tanto que si no se produce de forma eficiente y eficaz puede entrañar:

1. Escollos en el logro de aprendizaje significativo por causa de falta de motivación o recursos por parte de docentes, familias, e incluso el mismo estudiante.

2. Estrés para docentes y estudiantes a causa de la falta de certeza sobre el desenvolvimiento de los acontecimientos en la ejecución del acto pedagógico.

3. El aumento de inequidad en el acceso a haberes culturales entre estudiantes que acuden a centros de aprendizaje públicos o privados, así como aquellos que se encuentran ubicados en regiones distintas.

4. Recargo de responsabilidades adicionales en las mujeres como sujetos de cohesión en el hogar, al asumir la tutoría académica de su prole para complementar las asignaciones de los docentes.

5. Frustración en aquellos padres y representantes que no dominan el uso de las plataformas tecnológicas para ayudar a los educandos. 


\section{Journal of the Academy | $37 \mid$}

6. Reducción de las posibilidades de adquirir ciertos aprendizajes para los estudiantes que se encuentren dentro de grupos especialmente vulnerables.

7. Profundización de la brecha digital y educativa para aquellos educandos de grupos socioeconómicos con menores posibilidades financieras, pues de acuerdo a los recursos de este tipo con los que cuenten, se puede establecer un punto de partida que determinaría desigualdad respecto a los más aventajados, que se encuentran contrapuestos a los que tienen que comenzar con menos ventajas.

A pesar de todo ello, el establecimiento de la educación remota igualmente representa una oportunidad para el redimensionamiento de la planificación y el currículo, en miras a adaptarlos a situaciones de urgencia como la aquí tratada. Esto se podría configurar a través de la promoción de mayor interacción en el hogar como espacio para el aprendizaje y la incorporación de la familia como un factor de incidencia directa en el modelo educacional.

Si bien es cierto que la brecha tecnológica ha constituido para diversidad de personas un obstáculo en la materialización efectiva de su derecho a la educación, esto no implica que el haber atestiguado esta situación no conlleve el estudio de todo lo positivo que podría implementarse para el establecimiento de los correctivos adecuados. Es aquí como entra en la ecuación el Estado, pues a través de un análisis del escenario durante la pandemia y post pandemia, debería asumir que al adquirir compromisos jurídicos internacionales como los de la Declaración Universal de los Derechos Humanos, tiene la obligación de asegurar que se cumpla todo lo allí plasmado, y esto puede ser un logro posible por medio del redimensionamiento de las políticas públicas para establecer partidas presupuestarias que sirvan para fomentar el correcto acceso a servicios de conexión a lo largo y ancho de su territorio, e igualmente debería adecuar sus marcos jurídicos e incluso curriculares en concordancia con esas máximas estatuidas para la consolidación de una población que goce de integridad física, psíquica y moral.

Como progresión de todo lo explanado en este trabajo, igualmente podrían realizarse estudios de carácter comparativos que contrasten los contextos de la educación y las TIC, a través de la confrontación de los escenarios pre-pandemia, en pandemia y post pandemia, de manera que puedan elevarse propuestas para tratar de reducir las inequidades vistas en caso de generarse situaciones similares. También resultaría útil desarrollar investigaciones acerca de las 


\section{Journal of the Academy $|38|$}

implicaciones de la brecha digital para el ejercicio de otros derechos como el trabajo, libertad de expresión, entre otros.

\section{CONCLUSIONES}

Las emergencias que se suscitan dentro de las sociedades de acuerdo con el devenir del tiempo, muchas veces ponen sobre el tapete las debilidades que ellas tienen con respecto a los recursos con los cuales cuentan para la garantía de la vida digna que el Estado debe proteger con respecto a sus conformantes. Por ello fuera del aspecto negativo, una implicación positiva de este hecho es que representa una oportunidad para reconducir las carencias colectivas.

En concordancia con los objetivos planteados para el desarrollo de este artículo, se pudo concluir que: La brecha digital es la diferencia en el alcance que tienen las personas a las herramientas tecnológicas para la catálisis de acciones en el marco del siglo XXI, y en medio de la pandemia ha sacado a la luz la vulnerabilidad que existe entre los distintos estratos sociales, con arreglo a la mayor o menor medida en su acceso.

Además, debe destacarse que el derecho a la educación consiste en un derecho humano que conlleva la puerta hacia el aprendizaje, e igualmente puede catalizar la materialización de mejores oportunidades para lograr una vida digna. Es deber de los Estados asegurarlo en un mínimo que permita a las personas desarrollar destrezas para que no se encuentren desvalidas ante los retos actuales.

Asimismo, La brecha digital ha condicionado el ejercicio del derecho a la educación e igualmente ahonda esas diferencias porque de acuerdo a las oportunidades que tenga cada individuo, podría participar o no en el aprendizaje con absoluto aprovechamiento del acto educativo. Esto representa un problema en el contexto de la pandemia porque visto el fenómeno, muchos territorios de los diferentes continentes han tenido que asumir la pedagogía a distancia cuando en realidad no todos cuentan con los insumos para establecerla de forma óptima.

Si bien es cierto que el aislamiento prolongado ha representado un medio de restricción de lo que todos conocen como vida normal, también constituye un nuevo espacio de oportunidades para estudiar con mayor profundidad a consideraciones que antes no se tenían en cuenta si no 


\section{Journal of the Academy $|39|$}

causaban mayor afectación de manera individual. Por esto hay que tomar lo sucedido como un factor de impulso que puede provocar el inicio del cambio de paradigmas en la realización del acto educativo para que se ajuste a los nuevos tiempos.

\section{REFERENCIAS BIBLIOGRÁFICAS}

Alvarado, R., Díaz, C., Gómez, D. y Martínez, M. (2018). La brecha digital: una revisión conceptual y sus aportaciones metodológicas para su estudio en México. Entreciencias, 6(16), 49-64. http://dx.doi.org/10.22201/enes1.20078064e.2018.16.62611

Arias, A. y Chaves, H. (2010). Los Derechos Fundamentales contenidos en el marco Jurídico que regula las telecomunicaciones del País después de la promulgación del Tratado de Libre Comercio con Estados Unidos y sus leyes complementarias. Tesis de licenciatura. Costa Rica: Universidad de Costa Rica. https://tinyurl.com/y54zct7d

Banco de Desarrollo de América Latina (2020). Transformación digital para la América Latina del S. XXI, $\quad C A F . \quad$ España. <https://www.caf.com/es/conocimiento/visiones/2020/02/transformacion-digital-paralaamerica-latina-del-s21/> (22 de noviembre 2020).

Brito, A. (2015). Guía para la elaboración, corrección y asesoramiento de trabajos de investigación. San Tomé: Universidad Nacional Experimental Politécnica de la Fuerza Armada Bolivariana.

Centro de Estudios en Salud y Sociedad (2020). Dossier Covid 19 Impactos socioculturales de la pandemia. México: El Colegio de Sonora.

Duk, C. y Murillo, J. (2020). El Covid-19 y las Brechas Educativas. Revista latinoamericana de educación inclusiva, 14(1), 11-13. https://dx.doi.org/10.4067/S0718$\underline{73782020000100011}$

Dulzaides, M. y Molina, A. (2004). Análisis documental y de información: dos componentes de un mismo proceso. ACIMED, $12(2), \quad$ http://scielo.sld.cu/scielo.php?script=sci_arttext\&pid=S102494352004000200011\&lng =es\&tlng=es.

García, A. y González, A. (2013). Internet y África: de la brecha a la esperanza digital. Redes, libertades y comunicación. Revista científica en el ámbito de la comunicación aplicada, 3(2), 113-131. https://dialnet.unirioja.es/servlet/articulo?codigo=4467587

Henríquez, G. (2019). Venezuela tiene peor conexión a internet que Cuba e Iraq, Runrunes. España, Octubre 11. <https://runrun.es/noticias/390448/venezuela-tiene-peor-conexiona-internet-que-cuba-e-iraq/> (18 de noviembre 2020).

Horbath, J. E.; Gracia, M. A. (2016). El derecho a la educación: Un análisis a partir de la política educativa de las dos últimas décadas en México. Revista de Relaciones Internacionales, Estrategia y Seguridad, vol. 11(1), 171-191. https://www.redalyc.org/pdf/927/92743369009.pdf 


\section{Journal of the Academy $|40|$}

Martin, R., Moore, J y Schindler, S. (2016). Definiendo la desigualdad. ARQ (Santiago), (93), 30-41. https://dx.doi.org/10.4067/S0717-69962016000200005

Mendoza, L. (2020). Lo que la pandemia nos enseñó sobre la educación a distancia. Revista Latinoamericana de Estudios Educativos, $L$ (núm. esp.), 333-352. https://www.redalyc.org/jatsRepo/270/27063237028/html/index.html

Miguel, J. (2020). La educación superior en tiempos de pandemia: una visión desde dentro del proceso formativo. Revista Latinoamericana de Estudios Educativos, L (núm. esp.), 1340. https://rlee.ibero.mx/index.php/rlee/article/view/95

Muñoz Flores, J. (2010). El papel fundamental de Internet2 para el desarrollo de los entornos virtuales de aprendizaje y su impacto en la brecha digital. Revista mexicana de $\begin{array}{lll}\text { investigación 17-33. } & \text { 15(44), }\end{array}$ http://www.scielo.org.mx/scielo.php?script=sci_arttext\&pid=S1405$66662010000100003 \& \operatorname{lng}=$ es\&tlng=es.

Muñoz, V. (2014). El derecho humano a la educación. Sinéctica, (42), 1-10. http://www.scielo.org.mx/scielo.php?script=sci arttext\&pid=S1665109X2014000100007\&lng=es\&tlng=es.

Organización de Naciones Unidas (1948). Declaración Universal de los Derechos Humanos [página web]. ONU. 〈https://www.un.org/es/universal-declaration-human-rights/> (17 de noviembre 2020).

Organización de Naciones Unidas (1966). Pacto Internacional de Derechos Económicos, $\begin{array}{lllll}\text { Sociales } & y & \text { Culturales } & \text { [página }\end{array}$ <https://www.ohchr.org/SP/ProfessionalInterest/Pages/CESCR.asp> (17 de noviembre 2020)

Organización Panamericana de la Salud (2020). La OMS caracteriza a COVID-19 como una pandemia. Organización Panamericana de la Salud. $<$ https://www.paho.org/hq/index.php?option=com_content $\&$ view=article\&id=15756:w ho-characterizes-covid-19-as-a-pandemic\&Itemid=1926\&lang=es $>$ (15 de noviembre 2020).

Pizan-Campos, E., Barros-Sevillano, S. y Yupari-Azabache, I. (2020). Impacto del COVID-19 en la educación de los estudiantes de medicina del Perú. Revista de la Facultad de Medicina Humana, 20(3), 534-535. https://dx.doi.org/10.25176/rfmh.v20i3.2959

Ruiz, G. (2020). Covid-19: pensar la educación en un escenario inédito. Revista mexicana de investigación educativa, 25(85), 229-237. http://www.scielo.org.mx/scielo.php?script=sci_arttext\&pid=S140566662020000200229\&lng=es\&tlng=es.

Sánchez F. (2019). Fundamentos epistémicos de la investigación cualitativa y cuantitativa: Consensos y disensos. Revista Digital Investigación y Docencia 13 (1). 101-122. http://www.scielo.org.pe/scielo.php?pid=S222325162019000100008\&script=sci_arttex $\underline{\mathrm{t}}$ 


\section{Journal of the Academy $|41|$}

Sánchez, L., Reyes, A., Ortiz, D- y Olarte, F. (2017). El rol de la infraestructura tecnológica en relación con la brecha digital y la alfabetización digital en 100 instituciones educativas de Colombia. Calidad en la educación, (47), 112-144. https://dx.doi.org/10.4067/S0718$\underline{45652017000200112}$

Unicef (08 agosto, 2020). UNICEF entrega 240.000 guías de autoaprendizaje para impulsar la continuidad de la educación intercultural y bilingüe en Ecuador., Unicef. 8 de agosto. <https://www.unicef.org/ecuador/comunicados-prensa/unicef-entrega-240000-

gu\%C3\% ADas-de-autoaprendizaje-para-impulsar-la-continuidad-de-0 $>\quad(5 \quad$ de noviembre 2020).

Velásquez, R. (2020). La Educación Virtual en tiempos de Covid-19. Revista Científica Internacional, 3(1), 19-25. doi:https://doi.org/10.46734/revcientifica.v2i1.8 